\title{
Reduced description of nonequilibrium processes and correlation functions. Divergences and non-analyticity
}

\author{
A.I.Sokolovsky \\ Dnipropetrovs'k National University, 13, Naukova St., Dnipropetrovs'k, 49050, Ukraine
}

Received May 16, 2006, in final form July 6, 2006

\begin{abstract}
A complete theory for investigation of time correlation functions is developed on the basis of the Bogolyubov reduced description method proceeding from his functional hypothesis. The problem of convergence in the theory of nonequilibrium processes and its relation to the non-analytic dependence of basic values of the theory on a small parameter of the perturbation theory are discussed. A natural regularization of integral equations of the theory is proposed. In the framework of a model of slow variables (hydrodynamics of a fluid, kinetics of a gas) a generalized perturbation theory without divergencies is constructed corresponding to a partial summation in a usual perturbation theory. Properties of Green functions are discussed on the basis of resolvent formalism for the Liouville operator. A generalized Ernst and Dorfman theory is elaborated allowing to study the peculiarities of correlation and Green functions and to solve the convergence problem in the reduced description method.
\end{abstract}

Key words: reduced description of nonequilibriun processes, functional hypothesis, convergence problem, natural regularization, asymptotics of time correlation functions, Green functions, peculiarities of correlation and Green functions, generalized Ernst and Dorfman theory

PACS: $05.20 . D d, 05.30-d$

\section{Introduction}

The closely related Green functions and time correlation functions play an important role in condensed matter physics. From another viewpoint they are integrated within the structure of the theory of nonequilibrium processes. The problem of convergence of the obtained results is very important in all applications of this theory. In the kinetic theory it can be solved based on the few-body dynamics. However, in some cases the problem of convergence can be analyzed only based on the many particle dynamics. In this paper a generalization of the Ernst and Dorfman approach [1] (applied to the investigation of hydrodynamic problems by them) is discussed. In fact they proposed a theory for investigating the peculiarities of correlation functions based on Zwanzig [2], Mori [3] results and mode-mode coupling theory [4].

The principal method of modern theory of nonequilibrium processes is the Bogolyubov reduced description method. This method was developed by Bogolyubov [5] based on the functional hypothesis. This approach was widely elaborated by Peletminsky and coauthors (see [6]). The reduced description idea was also realized by Zubarev within the framework of his method of nonequilibrium statistical operator (see, for example, [7]). The application of the theory to the investigation of the Green and correlation functions was discussed in detail in the both approaches. However, the problem of convergence of the theory objects calculated in a perturbation theory has been investigated only to a small extent. Usually the divergence of a contribution of the perturbation theory speaks of non-analytical dependence of the considered value on a small parameter. Ernst and Dorfman demonstrated a fruitful approach to this problem in his studying the dispersion relation for a fluid [1]. In the present paper we propose a generalization of their approach based on the complete analysis of asymptotics of time correlation functions. In fact this is a general theory of investigation of peculiarities of correlation functions and corresponding Green functions. Therefore, it permits to study the convergence problem in the nonequilibrium theory.

The paper plan is as follows. 
The second section of the paper discusses the reduced description method based on the functional hypothesis. Here a time reversible system is studied, for which an evolution can be formally considered in both directions of time. This reflects a situation in the modern theory where fundamental mechanics is reversible. The observed nonequilibrium evolution in the future can be considered as a consequence of broken symmetry with respect to time reversal.

The third section discusses the reduced description close to equilibrium with application to investigating the asymptotics of time correlation functions. The investigation is based on the functional hypothesis and can be considered as a method of the initial state variation.

The forth section discusses the problem of regularization of integral equations of the theory and proposes a natural regularization procedure. It is more convenient than the Abel regularization because it does not introduce non-physical parameters in the theory. The regularized integral equation can be solved in a perturbation scheme that corresponds to a partial summation of the usual perturbation theory. The reduced description of a system with slow variables is considered as an important example. Within the framework of this model hydrodynamics of a fluid and kinetics of a gas can be investigated.

The fifth section discusses a connection of Green functions and time correlation functions within the framework of the formalism of the Liouville operator resolvent. A relation permitting to study the Green function peculiarities based on the elaborated procedure of calculation of time correlation function asymptotics is formulated.

The sixth section proposes the generalization of the Ernst and Dorfman approach which is formulated as a theory for investigating the peculiarities of correlation and Green functions.

The seventh section discusses the reduced description of a system taking into account nonequilibrium correlations as new independent reduced description parameters. A relation between the phenomenological and microscopic approaches is discussed.

The eighth section constructs the generalized Ernst and Dorfman theory taking into account binary correlations and predicts some corrections to their hydrodynamic theory.

In Conclusions we summarize the ways of investigating the peculiarities of correlation and Green functions and taking into account the results in the reduced description method.

\section{Reduced description of nonequilibrium states based on the functional hypothesis}

An arbitrary nonequilibrium state of a system is described by its nonequilibrium statistical operator $\rho(t)$ which satisfies the quantum Liouville equation

$$
\partial_{t} \rho(t)=\mathbf{L} \rho(t), \quad \mathbf{L} \rho \equiv-\frac{\mathrm{i}}{\hbar}[\hat{H}, \rho] .
$$

We shall discuss the properties of a time reversible system, for which it is possible to consider nonphysical evolution in the past too. So, we assume that at times $\pm t \gg \tau_{0}$ the system is described by parameters

$$
S p \rho(t) \hat{\eta}_{a} \underset{ \pm t \gg \tau_{0}}{\longrightarrow} \eta_{a}^{( \pm)}\left(t, \rho_{0}\right), \quad\left(\rho_{0} \equiv \rho(t=0)\right),
$$

where $\hat{\eta}_{a}$ are corresponding operators. The Bogolyubov functional hypothesis describes the structure of the statistical operator of the system at times $\pm t \gg \tau_{0}$ and has a form

$$
\rho(t) \underset{ \pm t \gg \tau_{0}}{\longrightarrow} \rho^{( \pm)}\left(\eta\left(t, \rho_{0}\right)\right),
$$

(the idea to consider the consequences of the time reversibility of a system was proposed in [8]). The asymptotic statistical operator $\rho^{( \pm)}\left(\eta\left(t, \rho_{0}\right)\right)$ is supposed to be an exact solution of the Liouville equation

$$
\partial_{t} \rho^{( \pm)}\left(\eta\left(t, \rho_{0}\right)\right)=\mathbf{L} \rho^{( \pm)}\left(\eta\left(t, \rho_{0}\right)\right), \quad\left( \pm t \gg \tau_{0}\right) .
$$

Equations (2), (3) show that the formula

$$
S p \rho^{( \pm)}\left(\eta^{( \pm)}\left(t, \rho_{0}\right)\right) \hat{\eta}_{a}=\eta^{( \pm)}\left(t, \rho_{0}\right), \quad\left( \pm t \gg \tau_{0}\right)
$$


is true and with (4) leads to the following generalized kinetic equation

$$
\partial_{t} \eta_{a}^{( \pm)}\left(t, \rho_{0}\right)=L_{a}^{( \pm)}\left(\eta^{( \pm)}\left(t, \rho_{0}\right)\right), \quad L_{a}^{( \pm)} \equiv S p \hat{\eta}_{a} \mathbf{L} \rho^{( \pm)}(\eta), \quad\left( \pm t \gg \tau_{0}\right)
$$

Reduced description parameters $\eta_{a}^{( \pm)}\left(t, \rho_{0}\right)$ as functions of $t$ and $\rho_{0}$ change in some domain $\mathcal{A}$, in which formula (5) gives

$$
\operatorname{Sp} \rho^{( \pm)}(\eta) \hat{\eta}_{a}=\eta_{a}, \quad\left(\eta_{a} \in \mathcal{A}\right)
$$

So, we can consider here $\eta_{a}$ as independent variables. Further, formulae (4), (5) lead to an equation for the statistical operator $\rho^{( \pm)}$

$$
\sum_{a} \frac{\partial \rho^{( \pm)}(\eta)}{\partial \eta_{a}} L_{a}^{( \pm)}(\eta)=\mathbf{L} \rho^{( \pm)}(\eta), \quad\left(\eta_{a} \in \mathcal{A}\right),
$$

which should be studied with equation (7) together. A remark that such a set of equations has two solutions belongs to Bogolyubov [5]. According to [5], one needs a boundary condition to select a proper solution. This condition should contain evolution in the corresponding direction of time.

It is important that a solution of the kinetic equation (6) can be continued for times $\pm t \geqslant 0$. This procedure introduces effective initial conditions $\eta_{a}^{( \pm)}\left(0, \rho_{0}\right)$ for kinetic equation $(6)$ and, besides, it makes equation (4) be valid for times $\pm t \geqslant 0$ too. In fact, the effective initial conditions were introduced by the Bogolyubov functional hypothesis. However, a detailed investigation of their calculation problem was performed by Grad [9] in his investigation of the hydrodynamics based on the Boltzmann equation.

For purposes of this paper it is enough to construct the mentioned boundary condition for the set of equations $(7),(8)$ based on the functional hypothesis (3) written in the form

$$
e^{t \mathbf{L}} \rho_{0} \underset{ \pm t \gg \tau_{0}}{\longrightarrow} e^{t \mathbf{L}} \rho^{( \pm)}\left(\eta^{( \pm)}\left(0, \rho_{0}\right)\right)
$$

(in fact, this idea was used in [6] to build hydrodynamics). Let us choose $\rho_{0}$ in the form of a quasi-equilibrium statistical operator $\rho_{q}(Y)$

$$
\rho(Y)=\exp \left\{\Omega(Y)-\sum_{a} Y_{a} \hat{\eta}_{a}\right\}, \quad S p \rho_{q}(Y)=1
$$

in order to construct the boundary condition based on the relation (9). Strictly speaking, let $\rho_{0} \rightarrow \rho_{q}\left(Y^{( \pm)}(\eta)\right)$ where $Y_{a}^{( \pm)}(\eta)$ is an inverse function for $\eta_{a}^{( \pm)}\left(0, \rho_{q}(Y)\right)$ and the following relation is valid

$$
\eta_{a}^{( \pm)}\left(0, \rho_{q}\left(Y^{( \pm)}(\eta)\right)\right)=\eta_{a}
$$

In this way, formula (9) takes a form

$$
e^{\tau \mathbf{L}}\left\{\rho_{q}\left(Y^{( \pm)}(\eta)\right)-\rho^{( \pm)}(\eta)\right\} \underset{ \pm \tau \gg \tau_{0}}{\longrightarrow} 0
$$

and gives the necessary boundary condition to equations $(7),(8)$. In some cases the following idea is very useful: relations

$$
f(\tau) \underset{ \pm \tau \gg \tau_{0}}{\longrightarrow} f^{( \pm)}, \quad f(0)=f^{( \pm)}-\int_{0}^{ \pm \infty} \mathrm{d} \tau \dot{f}(\tau)
$$

are equivalent (here the second relation can be considered as an integral form of the first one). The boundary condition (12) in the integral form and the Liouville equation (8) give an integral equation for the statistical operator $\rho^{( \pm)}(\eta)$

$$
\rho^{( \pm)}(\eta)=\rho_{q}\left(Y^{( \pm)}(\eta)\right)+\int_{0}^{ \pm \infty} \mathrm{d} \tau e^{\tau \mathbf{L}}\left\{\mathbf{L} \rho_{q}\left(Y^{( \pm)}(\eta)\right)-\sum_{a} \frac{\partial \rho^{( \pm)}(\eta)}{\partial \eta_{a}} L_{a}^{( \pm)}(\eta)\right\} .
$$

This equation together with (7) permits to calculate this operator $\rho^{( \pm)}(\eta)$ and auxiliary function $Y_{a}^{( \pm)}(\eta)$ for some models. 


\section{Reduced description close to equilibrium}

We restrict ourselves here to a model

$$
\begin{array}{cl}
\hat{\eta}_{a}^{+}=\hat{\eta}_{a} ; \quad & \hat{\dot{\eta}}_{a} \equiv-\mathbf{L} \hat{\eta}_{a} \sim \lambda, \quad \lambda \ll 1 ; \\
\rho_{q}(\stackrel{o}{Y})=w, \quad \stackrel{o}{Y}_{a} \equiv Y_{a}^{( \pm)}(\stackrel{o}{\eta}) ; \quad \stackrel{o}{a}_{a} \equiv S p w \hat{\eta}_{a},
\end{array}
$$

where $w$ is an equilibrium statistical operator of the system, $\stackrel{o}{\eta}_{a}$ are equilibrium values of variables $\eta_{a}$. Within the framework of this model, for example, hydrodynamics of a fluid and kinetics of a gas can be considered.

For states which are close to equilibrium the statistical operator of the system and reduced description parameters have a form

$$
\rho_{0}=w+\delta \rho_{0}+O\left(g^{2}\right), \quad \eta_{a}^{( \pm)}\left(t, \rho_{0}\right)=\stackrel{o}{\eta}_{a}+\delta \eta_{a}^{( \pm)}\left(t, \delta \rho_{0}\right)+O\left(g^{2}\right)
$$

The value $g$ is a small parameter and $\delta \rho_{0} \sim g, \delta \eta_{a}^{( \pm)}\left(t, \delta \rho_{0}\right) \sim g$. The initial statistical operator of the system $\rho_{0}$ should satisfy the Bogolyubov principle of spatial correlation weakening (see, for example, [6]). Therefore, the operator $\delta \rho_{0}$ can be chosen in the form [6]

$$
\delta \rho_{0}=w \chi(w, \hat{A}), \quad \chi(\rho, \hat{A}) \equiv \int_{0}^{1} \mathrm{~d} \xi \rho^{-\xi}\{\hat{A}-S p \rho \hat{A}\} \rho^{\xi},
$$

where $\hat{A}$ is an additive operator. In this situation (close to equilibrium) the formula

$$
\rho^{( \pm)}\left(\eta^{( \pm)}\left(t, \rho_{0}\right)\right)=w+\delta \rho^{( \pm)}(t)+O\left(g^{2}\right), \quad \delta \rho^{( \pm)}(t) \equiv \sum_{a} \rho_{a}^{( \pm)} \delta \eta_{a}^{( \pm)}\left(t, \delta \rho_{0}\right)
$$

with

$$
\rho^{( \pm)}(\stackrel{o}{\eta})=w,\left.\quad \rho_{a}^{( \pm)} \equiv \frac{\partial \rho^{( \pm)}(\eta)}{\partial \eta_{a}}\right|_{\eta=\eta}
$$

is true. According to (6), the functions $\delta \eta_{a}^{( \pm)}\left(t, \delta \rho_{0}\right)$ satisfy the equation

$$
\partial_{t} \delta \eta_{a}^{( \pm)}\left(t, \delta \rho_{0}\right)=\sum_{b} M_{a b}^{( \pm)} \delta \eta_{b}^{( \pm)}\left(t, \delta \rho_{0}\right), \quad( \pm t \geqslant 0),
$$

where

$$
M_{a b}^{( \pm)}=\operatorname{Sp} \hat{\dot{\eta}}_{a} \rho_{b}^{( \pm)}, \quad \operatorname{Sp} \rho_{a}^{( \pm)} \hat{\eta}_{b}=\delta_{a b}
$$

Now the functional hypothesis (3) and equation (20) give the following asymptotic relation

$$
e^{t \mathbf{L}} \delta \rho_{0} \underset{ \pm t \gg \tau_{0}}{\longrightarrow} \sum_{a, b} \rho_{a}^{( \pm)} e_{a b}^{t M^{( \pm)}} \delta \eta_{b}^{( \pm)}\left(0, \delta \rho_{0}\right)
$$

In hydrodynamics a similar relation was obtained in [6] for the real evolution and in [10] for evolution in the both directions of time. However, in [6,10] formula a kind of $(22)$ was discussed in the terms of variables connected with objects of the reduced description in a more complicated way than above. For kinetics, a relation similar to (22) was discussed in [8].

Choosing here the statistical operator $\delta \rho_{0}$ in the form (17) and using definitions of quantum correlation function and equilibrium time correlation function $K_{A B}(t)$

$$
(\hat{A}, \hat{B})=\operatorname{Sp} \hat{A} w \chi(w, \hat{B}), \quad K_{A B}(t)=\left(\hat{A}, e^{t \mathbf{L}} \hat{B}\right),
$$


we obtain a general formula for asymptotics of equilibrium time correlation functions

$$
K_{A B}(t) \underset{ \pm t \gg \tau_{0}}{\longrightarrow} K_{A B}^{( \pm)}(t), \quad K_{A B}^{( \pm)}(t) \equiv \sum_{a, b} \operatorname{Sp} \hat{A} \rho_{a}^{( \pm)} e_{a b}^{t M^{( \pm)}} \mathbf{C}_{b}^{( \pm)}(\hat{B})
$$

Here the value $\mathbf{C}_{b}^{( \pm)}(\hat{A})$ is defined by the formula

$$
\mathbf{C}_{a}^{( \pm)}(\hat{A})=\delta \eta_{a}^{( \pm)}(0, w \chi(w, \hat{A}))
$$

and is called the memory function hereafter (this function was introduced in [11]). Calculation of these functions can be simplified based on the formula

$$
\mathbf{C}_{a}^{( \pm)}(\hat{A})=\sum_{b} \operatorname{Sp} \hat{A} \rho_{b}^{(\mp)} A_{b a}^{(\mp)}, \quad A_{a b}^{( \pm)} \equiv \mathbf{C}_{a}^{( \pm)}\left(\hat{\eta}_{b}\right) .
$$

Applying the relation (24) to the identity

$$
K_{A B}(t)=K_{B A}(-t)
$$

leads to the formula

$$
K_{A B}^{(+)}(t)=K_{B A}^{(-)}(-t), \quad(t \geqslant 0) .
$$

This relation gives the identity

$$
\sum_{a} \operatorname{Sp} \hat{A} \rho_{a}^{(+)} \mathbf{C}_{a}^{(+)}(\hat{B})=\sum_{a} \operatorname{Sp} \hat{B} \rho_{a}^{(-)} \mathbf{C}_{a}^{(-)}(\hat{A}),
$$

from which the necessary expression (26) follows with the help of the second formula in (21). Note that the idea of the above proof of the formula (26) belongs to Peletminsky [10].

The corresponding formula for correlation function of the reduced description parameters

$$
K_{a b}(t) \equiv\left(\hat{\eta}_{a}, e^{t \mathbf{L}} \hat{\eta}_{b}\right), \quad K_{a b}(t) \underset{ \pm t \gg \tau_{0}}{\longrightarrow} K_{a b}^{( \pm)}(t), \quad K_{a b}^{( \pm)}(t) \equiv \sum_{c} e_{a c}^{t M^{( \pm)}} A_{c b}^{( \pm)}
$$

which follows from (21) and (26) is an important particular case of the relation (24). This formula was discussed in [8] and was applied for defining kinetic coefficients in kinetics beyond the perturbation theory.

An integral equation for the statistical operator $\rho_{a}^{( \pm)}$can be obtained from its definition (19) and equation (14)

$$
\rho_{a}^{( \pm)}=\rho_{a}^{q( \pm)}+\int_{0}^{ \pm \infty} \mathrm{d} \tau e^{\tau \mathbf{L}}\left\{\mathbf{L} \rho_{a}^{q( \pm)}-\sum_{b} \rho_{b}^{( \pm)} M_{b a}^{( \pm)}\right\},
$$

where the quasi-equilibrium statistical operator is given by the expression

$$
\rho_{a}^{q( \pm)}=\sum_{b} w_{b} A_{b a}^{( \pm)-1}, \quad w_{a} \equiv w \chi\left(w, \hat{\eta}_{a}\right) .
$$

This operator contains matrix $A_{a b}^{( \pm)}$introduced by formula (26) because the formula

$$
\left.\frac{\partial Y_{a}^{( \pm)}(\eta)}{\partial \eta_{b}}\right|_{\eta=\eta}=-A_{a b}^{( \pm)-1}
$$

is valid according to (11). Integral equation (31) should be solved together with relations (21) with respect to $\rho_{a}^{( \pm)}, A_{a b}^{( \pm)}$and $M_{a b}^{( \pm)}$. Of course, equation (31) can be obtained directly from the Liouville equation at a reduced description

$$
\sum_{b} \rho_{b}^{( \pm)} M_{b a}^{( \pm)}=\mathbf{L} \rho_{a}^{( \pm)}
$$

and a boundary condition

$$
e^{\tau \mathbf{L}}\left\{\rho_{a}^{q( \pm)}-\rho_{a}^{( \pm)}\right\} \underset{ \pm \tau \gg \tau_{0}}{\longrightarrow} 0,
$$

which follows from (8) and (12). 


\section{Perturbation theory with partial summation and regularization of integral equations}

A resolvent operator

$$
R(z)=(z-\mathbf{L})^{-1},
$$

which enters the integral equations (14), (31) in the form of $R( \pm 0)$ because of the identity

$$
\int_{0}^{ \pm \infty} \mathrm{d} \tau e^{\tau \mathbf{L}} e^{-\tau z}=R(z \pm 0)
$$

plays an important role in our discussion. It is known that the operator $R( \pm \varepsilon)$ has a peculiarity at $\varepsilon \rightarrow 0$ (see, for example, [12]) and $R( \pm 0)$ exists only on the integrands in equations (14) and (31). A guarantee of the existence of $R( \pm 0)$ on a summand of the integrands and on a contribution to the integrands obtained in a perturbation theory does not exist. The latter is possible because basic values of the reduced description should not be regular functions of a small parameter of the theory $\lambda$.

In this situation the Abel regularization of integral equations is widely used in order to make an investigation possible. In this way, the equations

$$
\rho^{( \pm)}(\varepsilon)=\int_{0}^{ \pm \infty} \mathrm{d} \tau e^{\mp \tau \varepsilon} e^{\tau \mathbf{L}} \mathcal{K}\left(\rho^{( \pm)}\right) \quad \text { or } \quad \rho^{( \pm)}(\varepsilon)=R( \pm \varepsilon) \mathcal{K}\left(\rho^{( \pm)}\right)
$$

are used instead of equations of the form

$$
\rho^{( \pm)}=\int_{0}^{ \pm \infty} \mathrm{d} \tau e^{\tau \mathbf{L}} \mathcal{K}\left(\rho^{( \pm)}\right) \quad \text { or } \quad \rho^{( \pm)}=R( \pm 0) \mathcal{K}\left(\rho^{( \pm)}\right),
$$

$(\mathcal{K}(\rho)$ is a function) because

$$
\rho^{( \pm)}=\lim _{\varepsilon \rightarrow+0} \rho^{( \pm)}(\varepsilon) .
$$

Note in this connection that in a general case

$$
\rho^{( \pm)} \neq \lim _{\varepsilon \rightarrow+0} \rho_{1}^{( \pm)}(\varepsilon), \quad \rho_{1}^{( \pm)}(\varepsilon) \equiv \int_{0}^{ \pm \infty} \mathrm{d} \tau e^{\mp \tau \varepsilon} e^{\tau \mathbf{L}} \mathcal{K}\left(\rho_{1}^{( \pm)}(\varepsilon)\right),
$$

because this approach leads to the products of the form $\left(a \varepsilon^{-1}+b \varepsilon+\cdots\right)(m+n \varepsilon+\cdots)$ with incorrect contribution an to $\rho^{( \pm)}$.

It is much more convenient to use a natural regularization of the discussed integral equation (31) which corresponds to a partial summation of a series of the perturbation theory based on (15). In order to construct the regularized integral equation, note that in the leading approximation in $\lambda$, a solution of equation (31) is given by formulae

$$
\rho_{a}^{( \pm)(0)}=\sum_{b} w_{b} K_{b a}^{-1}, \quad A_{a b}^{( \pm)(0)}=K_{a b}, \quad M_{a b}^{( \pm)(1)}=\stackrel{o}{M} a b,
$$

where

$$
K_{a b}=\left(\hat{\eta}_{a}, \hat{\eta}_{b}\right), \quad \stackrel{o}{M} a b=\sum_{c}\left(\hat{\dot{\eta}}_{a}, \hat{\eta}_{c}\right) K_{c b}^{-1}
$$

Let us introduce right and left eigenvectors of the matrix $\stackrel{o}{M} a$

$$
\sum_{b} \stackrel{o}{M_{a b}} \chi_{n b}=\mathrm{i} \omega_{n} \chi_{n a}, \quad \sum_{a} \psi_{n a} \stackrel{o}{M} a b=\mathrm{i} \omega_{n} \psi_{n b}
$$


It is easy to prove that

$$
\omega_{n}^{*}=\omega_{n}, \quad \psi_{n a}=\sum_{b} \chi_{n b}^{*} K_{b a}^{-1}, \quad \psi_{\sigma(n) a}=\psi_{n a}^{*}, \quad \omega_{\sigma(n)}=-\omega_{n}, \quad K_{a b}^{*}=K_{a b},
$$

where $\sigma(n)$ is a function defined on the numbers of eigenvectors. In this way, the following properties of the correlation function (23)

$$
(\hat{A}, \hat{B})=(\hat{B}, \hat{A}), \quad(\hat{A}, \hat{B})^{*}=\left(\hat{A}^{+}, \hat{B}^{+}\right), \quad(\mathbf{L} \hat{A}, \hat{B})=-(\hat{A}, \mathbf{L} \hat{B})
$$

are useful. Note that the introduction of the eigenvectors and eigenvalues with (44) is slightly schematic because, for example, in hydrodynamics and kinetics these values depend on a wave vector as a parameter.

A contribution $\stackrel{o}{M}_{a b}$ in $M_{a b}$ describes a reversible evolution of the system because its eigenvalues $i \omega_{n}$ are imaginary. Therefore, the boundary condition (35) can be rewritten in the form

$$
e^{\tau \mathbf{L}} \sum_{b}\left\{\rho_{b}^{q( \pm)}-\rho_{b}^{( \pm)}\right\} e_{b a}^{\tau \stackrel{o}{M}} \underset{ \pm \tau \gg \tau_{0}}{\longrightarrow} 0, \quad\left(e_{a b}^{\tau \stackrel{o}{M}} \equiv\left(e^{\tau \stackrel{o}{M}}\right)_{a b}\right)
$$

The boundary condition (47) in the integral form and the Liouville equation (34) give a new integral equation for the statistical operator $\rho_{a}^{( \pm)}$

$\rho_{a}^{( \pm)}=\rho_{a}^{q( \pm)}+\int_{0}^{ \pm \infty} \mathrm{d} \tau e^{\tau \mathbf{L}} \sum_{b}\left\{\mathbf{L} \rho_{b}^{q( \pm)}-\sum_{c} \rho_{c}^{q( \pm)} M_{c b}^{( \pm)}-\sum_{c}\left(\rho_{c}^{( \pm)}-\rho_{c}^{q( \pm)}\right)\left(M_{c b}^{( \pm)}-\stackrel{o}{M} c b\right)\right\} e_{b a}^{-\tau \stackrel{o}{M}}$

which should be solved together with relations (21) with respect to $\rho_{a}^{( \pm)}, A_{a b}^{( \pm)}$and $M_{a b}^{( \pm)}$. This equation is a naturally regularized integral equation (31). The idea of this regularization and equation (48) were proposed in [13] for hydrodynamics.

In the developed theory it is very convenient to choose linear combinations

$$
\hat{\eta}_{n}=\sum_{a} \psi_{n a} \hat{\eta}_{a}, \quad \hat{\eta}_{a}=\sum_{n} \chi_{n a} \hat{\eta}_{n}
$$

instead of variables $\hat{\eta}_{a}$. For this case equations (48), (32) and (21) take the same form and can be written using simple substitution $a, b, c \rightarrow n, n^{\prime}, n^{\prime \prime}$. However, additional simplifications are possible because

$$
\hat{\eta}_{n}^{+}=\hat{\eta}_{\sigma(n)}, \quad K_{n \sigma\left(n^{\prime}\right)}=\delta_{n n^{\prime}} \quad \text { or } \quad\left(\hat{\eta}_{n}, \hat{\eta}_{n^{\prime}}^{+}\right)=\delta_{n n^{\prime}} ; \quad \stackrel{o}{M_{n n^{\prime}}}=\mathrm{i} \omega_{n} \delta_{n n^{\prime}}
$$

according to (44), (45), and (49). The last formula means that variables $\delta \eta_{n}^{( \pm)}\left(t, \delta \rho_{0}\right)$ are the modes of the system with respect to evolution with matrix $\stackrel{o}{M}$ ab. Using these results and the relation (37), one can rewrite the integral equation (48) in the form

$$
\rho_{n}^{( \pm)}=\rho_{n}^{q( \pm)}+R\left(\mathrm{i} \omega_{n} \pm 0\right)\left\{\mathbf{L} \rho_{n}^{q( \pm)}-\sum_{n^{\prime}} \rho_{n^{\prime}}^{q( \pm)} M_{n^{\prime} n}^{( \pm)}-\sum_{n^{\prime}}\left(\rho_{n^{\prime}}^{( \pm)}-\rho_{n^{\prime}}^{q( \pm)}\right)\left(M_{n^{\prime} n}^{( \pm)}-\mathrm{i} \omega_{n} \delta_{n^{\prime} n}\right)\right\}
$$

with a following expression for the quasi-equilibrium statistical operator

$$
\rho_{n}^{q( \pm)}=\sum_{n^{\prime}} w_{n^{\prime}} A_{n^{\prime} n}^{( \pm)-1}, \quad w_{n} \equiv w \chi\left(w, \hat{\eta}_{n}\right)
$$

and additional equations

$$
M_{n n^{\prime}}^{( \pm)}=\operatorname{Sp} \hat{\dot{\eta}}_{n} \rho_{n^{\prime}}^{( \pm)}, \quad \operatorname{Sp} \hat{\eta}_{n} \rho_{n^{\prime}}^{( \pm)}=\delta_{n n^{\prime}}
$$


Equation (51) is solvable in a generalized perturbation theory in $\lambda$, which is based on the model (15) without expanding the resolvent operator $R\left(\mathrm{i} \omega_{n} \pm 0\right)$ in powers of $\omega_{n}$ (despite $\left.\omega_{n} \sim \lambda\right)$. It is obvious that this theory corresponds to a partial summation within the framework of the simple perturbation theory in $\lambda$.

The basic formula (24) for asymptotic of the time correlation functions leads now to the expressions

$$
K_{A B}^{( \pm)}(t)=\sum_{n n^{\prime}} \operatorname{Sp} \hat{A} \rho_{n}^{( \pm)} e_{n n^{\prime}}^{t M^{( \pm)}} \mathbf{C}_{n^{\prime}}^{( \pm)}(\hat{B}), \quad \mathbf{C}_{n}^{( \pm)}(\hat{A})=\sum_{n^{\prime}} \operatorname{Sp} \hat{A} \rho_{n^{\prime}}^{(\mp)} A_{n^{\prime} n}^{(\mp)}
$$

\section{Green functions and the reduced description method}

Equilibrium time correlation functions (23) and Green functions

$$
G_{A B}^{( \pm)}(t) \equiv \mp \frac{\mathrm{i}}{\hbar} \theta( \pm t) \operatorname{Sp} w[\hat{A}(t), \hat{B}], \quad \hat{A}(t) \equiv e^{-t \mathbf{L}} \hat{A}
$$

play an important role in condensed matter theory. The Fourier transformation $G_{A B}^{( \pm)}(\omega)$ of the Green function can be expressed through the corresponding correlation function by the formula

$$
G_{A B}^{( \pm)}(\omega)=\mathrm{i} \omega \beta \tilde{K}_{A B}(-\mathrm{i} \omega \pm 0)+\beta(\hat{A}, \hat{B}), \quad G_{A B}^{( \pm)}(\omega) \equiv \int_{-\infty}^{+\infty} \mathrm{d} t G_{A B}^{( \pm)}(t) e^{\mathrm{i} \omega t}
$$

where $\beta$ is reverse temperature of the considered equilibrium state of the system, $\tilde{K}_{A B}(z)$ is a matrix element of the resolvent operator

$$
\tilde{K}_{A B}(z)=(\hat{A}, R(z) \hat{B})
$$

Expression (56) follows from the relation (37), notation (17) and the identity

$$
\frac{\mathrm{i}}{\hbar}[w, \hat{B}(-t)]=-\beta \partial_{t} w \chi(w, \hat{B}(-t))
$$

(the idea of this transformation was used in [10] too).

An investigation of the peculiarities of the Green functions in this way is equivalent to studying the correlation functions $\tilde{K}_{A B}(z \pm 0)$. According to $(23),(37)$, and (54), the latter can be done using the relation

$$
\tilde{K}_{A B}(z \pm 0)=S_{A B}^{( \pm)}(z)+\tilde{K}_{A B}^{( \pm)}(z)
$$

where

$$
\begin{aligned}
S_{A B}^{( \pm)}(z) & =\int_{0}^{ \pm \infty} \mathrm{d} t\left\{K_{A B}(t)-K_{A B}^{( \pm)}(t)\right\} e^{-z t}, \\
\tilde{K}_{A B}^{( \pm)}(z) & \equiv \sum_{n n^{\prime}} \operatorname{Sp} \hat{A} \rho_{n}^{( \pm)}\left(z-M^{( \pm)}\right)_{n n^{\prime}}^{-1} \mathbf{C}_{n^{\prime}}^{( \pm)}(\hat{B}) .
\end{aligned}
$$

The asymptotic relation $(24)$ shows that $S_{A B}^{( \pm)}(z)$ is a regular function of $z$ and all peculiarities of $\tilde{K}_{A B}(z \pm 0)$ are related to the second term in (59). 


\section{Generalized Ernst and Dorfman approach to the investigation of asymptotics of correlation functions}

Let us calculate the basic values $\rho_{n}^{( \pm)}, A_{n n^{\prime}}^{( \pm)}, M_{n n^{\prime}}^{( \pm)}$of the theory for the states which are close to equilibrium in the generalized perturbation theory. Simple consideration gives

$$
\begin{aligned}
\rho_{n}^{( \pm)} & =w_{n}^{+}-w \chi\left(w, \mathbf{Q} R\left(\mathrm{i} \omega_{n} \pm 0\right) \hat{I}_{n}^{+}\right)+\ldots, \\
A_{n n^{\prime}}^{( \pm)} & =K_{n n^{\prime}}-\left(\hat{\eta}_{n}, R\left(-\mathrm{i} \omega_{n^{\prime}} \pm 0\right) \hat{I}_{n^{\prime}}\right)+\ldots, \\
M_{n n^{\prime}}^{( \pm)} & =\mathrm{i} \omega_{n} \delta_{n n^{\prime}}-\left(\hat{I}_{n}, R\left(i \omega_{n^{\prime}} \pm 0\right) \hat{I}_{n^{\prime}}^{+}\right)+\ldots,
\end{aligned}
$$

where the Mori projection operator $\mathbf{P}$ and values $\hat{I}_{n}$

$$
\mathbf{P} \hat{A}=\operatorname{Sp} w \hat{A}+\sum_{n}\left(\hat{\eta}_{n}-\stackrel{o}{\eta}_{n}\right) \operatorname{Sp} \hat{A} w_{n}^{+}, \quad \mathbf{Q}=1-\mathbf{P} ; \quad \hat{I}_{n} \equiv \mathbf{Q} \hat{\dot{\eta}}_{n}
$$

are supposed to be introduced. Definition (17) of the function $\chi(\rho, \hat{A})$ was taken into account too. The Mori projection operator has the following properties

$$
(\mathbf{P} \hat{A})^{+}=\mathbf{P} \hat{A}^{+}, \quad(\mathbf{P} \hat{A}, \hat{B})=(\hat{B}, \mathbf{P} \hat{A})
$$

which were used in (61). In hydrodynamics $\hat{I}_{n}$ can be considered as operators related to dissipative fluxes. Calculation of the memory function $\mathbf{C}_{n}^{( \pm)}(\hat{A})$ according to (54), (61), and (45) gives

$$
\mathbf{C}_{n}^{( \pm)}(\hat{A})=\left(\hat{\eta}_{n}, \hat{A}\right)+\left(\hat{I}_{n}, R\left(i \omega_{n} \pm 0\right) \hat{A}\right)+\cdots
$$

where the relation

$$
(\hat{A}, R(z) \hat{B})=-(\hat{B}, R(-z) \hat{A})
$$

was applied.

So, in the considered approximation the asymptotics (54) of the equilibrium time correlation function (24) is given by the formula

$$
K_{A B}^{( \pm)}(t)=\sum_{n n^{\prime}}\left\{\left(\hat{A}, \hat{\eta}_{n}^{+}\right)-\left(\mathbf{Q} \hat{A}, R\left(\mathrm{i} \omega_{n} \pm 0\right) \hat{I}_{n}^{+}\right)\right\} e_{n n^{\prime}}^{t M^{( \pm)}}\left\{\left(\hat{\eta}_{n^{\prime}}, \hat{B}\right)+\left(\hat{I}_{n^{\prime}}, R\left(\mathrm{i} \omega_{n^{\prime}} \pm 0\right) \hat{B}\right)\right\}
$$

Respectively, the function $\tilde{K}_{A B}(z)$ which defines the peculiarities of correlation functions (see (59), $(60))$ and the Green functions (see (56)) takes the form

$$
\begin{aligned}
\tilde{K}_{A B}^{( \pm)}(z)= & \sum_{n n^{\prime}}\left\{\left(\hat{A}, \hat{\eta}_{n}^{+}\right)-\left(\mathbf{Q} \hat{A}, R\left(\mathrm{i} \omega_{n} \pm 0\right) \hat{I}_{n}^{+}\right)\right\} \\
& \times\left[z-M^{( \pm)}\right]_{n n^{\prime}}^{-1}\left\{\left(\hat{\eta}_{n^{\prime}}, \hat{B}\right)+\left(\hat{I}_{n^{\prime}}, R\left(\mathrm{i} \omega_{n^{\prime}} \pm 0\right) \hat{B}\right)\right\}
\end{aligned}
$$

The next main task is to calculate the asymptotics of the obtained values at $\lambda \rightarrow 0$. A difficulty of this calculation consists in a possibility for the correlation functions not to be regular functions of $\lambda$. It is possible to start this investigation from matrix $M_{n n^{\prime}}$ which defines the evolution of the reduced description parameters $\delta \eta_{n}\left(t, \delta \rho_{0}\right)$. According to (57), in the considered approximation (61) this matrix is expressed through the function

$$
D_{n n^{\prime}, n^{\prime \prime}}^{( \pm)} \equiv D_{n n^{\prime}}\left(\mathrm{i} \omega_{n^{\prime \prime}} \pm 0\right), \quad D_{n n^{\prime}}(z) \equiv \tilde{K}_{I_{n} I_{n^{\prime}}^{+}}(z)
$$


Applying the relations (59) and (67) to this function gives the following set of equations for $M_{n n^{\prime}}^{( \pm)}$ and $D_{n n^{\prime}, n^{\prime \prime}}^{( \pm)}$

$$
\begin{aligned}
D_{n_{1} n_{2}, n^{\prime \prime}}^{( \pm)} & =S_{n_{1} n_{2}}^{( \pm)}\left(\mathrm{i} \omega_{n^{\prime \prime}}\right)-\sum_{n n^{\prime}} D_{n_{1} n, n}^{( \pm)}\left[\mathrm{i} \omega_{n^{\prime \prime}}-M^{( \pm)}\right]_{n n^{\prime}}^{-1} D_{n^{\prime} n_{2}, n^{\prime}}^{( \pm)} \\
M_{n n^{\prime}}^{( \pm)} & =\mathrm{i} \omega_{n} \delta_{n n^{\prime}}-D_{n n^{\prime}, n^{\prime}}^{( \pm)}
\end{aligned}
$$

where $S_{n n^{\prime}}^{( \pm)}(z) \equiv S_{I_{n} I_{n^{\prime}}^{+}}^{( \pm)}(z)$. These relations can be considered as generalized Ernst and Dorfman equations [1]. This equation discussion needs the function $S_{n_{1} n_{2}}^{( \pm)}\left(\mathrm{i} \omega_{n_{3}}\right)$ being estimated. In fact, in the hydrodynamic investigation in [1] this function is regarded to have schematically the structure $a k^{2}+O\left(k^{3}\right)$ and cannot effect the calculations.

Solving this equation with respect to $D_{n n^{\prime}, n^{\prime \prime}}^{( \pm)}$permits to investigate the correlation function (68) using the formula

$$
D_{n_{1} n_{2}}(z \pm 0)=S_{n_{1} n_{2}}^{( \pm)}(z)-\sum_{n n^{\prime}} D_{n_{1} n, n}^{( \pm)}\left[z-M^{( \pm)}\right]_{n n^{\prime}}^{-1} D_{n^{\prime} n_{2}, n^{\prime}}^{( \pm)}
$$

and to calculate asymptotics

$$
K_{I_{n_{1} I_{n_{2}}}^{( \pm)}}^{(t)}=-\sum_{n n^{\prime}} D_{n_{1} n, n}^{( \pm)} e_{n n^{\prime}}^{t M^{( \pm)}} D_{n^{\prime} n_{2}, n^{\prime}}^{( \pm)}
$$

(see (59), (67), and (66)).

The investigation of the correlation function $\tilde{K}_{A^{d} I_{n}^{+}}(z) \equiv E_{A n}(z)\left(\hat{A}^{d} \equiv \mathbf{Q} \hat{A}\right)$ can be performed based on the relations

$$
\begin{aligned}
E_{A n_{1}}(z \pm 0) & =S_{A^{d} I_{n_{1}}^{+}}^{( \pm)}(z)+\sum_{n n^{\prime}} E_{A I_{n}}\left(\mathrm{i} \omega_{n} \pm 0\right)\left(z-M^{( \pm)}\right)_{n n^{\prime}}^{-1} D_{n^{\prime} n_{1}, n^{\prime}}^{( \pm)} \\
E_{A n_{1}}\left(\mathrm{i} \omega_{n} \pm 0\right) & =S_{A^{d} I_{n_{1}}^{+}}\left(\mathrm{i} \omega_{n}\right)+\sum_{n n^{\prime}} E_{A n}\left(\mathrm{i} \omega_{n} \pm 0\right)\left(\mathrm{i} \omega_{n}-M^{( \pm)}\right)_{n n^{\prime}}^{-1} D_{n^{\prime} n_{1}, n^{\prime}}^{( \pm)}
\end{aligned}
$$

following from (59). A correlation function $F_{n A}(z) \equiv \tilde{K}_{I_{n} A^{+d}}(z)$ can be studied analogously. According to (59) and (67), functions $D_{n n^{\prime}}(z), E_{A n}(z), F_{n A}(z)$ define the behavior of the general correlation function $\tilde{K}_{A B}(z)$. This gives a complete information on the behavior of the Green function (56).

\section{Reduced description taking into account correlations as independent variables}

The fundamental idea of modern statistical mechanics is: consideration of the dynamics with random initial conditions reproduces all nonequilibrium states based on this dynamics. For example, the quantum (classical) Liouville equation describes all nonequilibrium states of a system and is based on quantum (classical) mechanics with random initial conditions. This idea can be applied fruitfully to equations of the reduced description method in order to take into account fluctuations $($ see $[14,15])$. Hereafter we give a generalization of this approach.

We shall consider the reduced description of a system with parameters introduced in section 2 . In order to simplify the notation, the evolution in the physical direction of time is investigated and superscript $(+)$ is dropped in formulae. Our starting point is the solution of the Cauchy problem $\eta_{a}\left(t, \eta_{0}\right)$ for kinetic equation (6)

$$
\partial_{t} \eta_{a}\left(t, \eta_{0}\right)=L_{a}\left(\eta\left(t, \eta_{0}\right)\right), \quad \eta_{a}\left(t=0, \eta_{0}\right) \equiv \eta_{a 0}
$$


Initial values of the reduced description parameters $\eta_{a 0}$ are considered as random values. Therefore, fluctuations (moments) of parameters $\eta_{a}\left(t, \eta_{0}\right)$ can be considered

$$
f_{a_{1} \ldots a_{s}}(t)=\overline{\eta_{a_{1}}\left(t, \eta_{0}\right) \ldots \eta_{a_{s}}\left(t, \eta_{0}\right)}, \quad f_{a}(t) \equiv \eta_{a}(t) .
$$

The investigation of the set of these variables is simplified by a generating functional

$$
\mathcal{F}(f(t), u)=\sum_{s=0}^{\infty} \frac{1}{s !} \sum_{a_{1} \ldots a_{s}} u_{a_{1}} \ldots u_{a_{s}} f_{a_{1} \ldots a_{s}}(t)=\overline{\exp \left\{\sum_{a} u_{a} \eta\left(t, \eta_{0}\right)\right\}}
$$

where $u_{a}$ are auxiliary values. Formulae (73) and (75) give the following equation for the generating functional

$$
\partial_{t} \mathcal{F}(f(t), u)=\left.\mathcal{F}\left(f(t), u+\frac{\partial}{\partial \eta}\right) \sum_{a} u_{a} L_{a}(\eta)\right|_{\eta=0},
$$

(for the case of hydrodynamics this equation was derived in [14]). Instead of the moments of reduced description parameters it is useful to introduce the centered moments (correlations) $g_{a_{1} \ldots a_{s}}(t)$ by the formulae

$$
\begin{aligned}
& \mathcal{F}(f(t), u)=\exp \left\{\sum_{a} u_{a} \eta_{a}(t)+\mathcal{G}(g(t), u)\right\}, \\
& \mathcal{G}(g(t), u)=\sum_{s=2}^{\infty} \frac{1}{s !} \sum_{a_{1} \ldots a_{s}} u_{a_{1}} \ldots u_{a_{s}} g_{a_{1} \ldots a_{s}}(t) .
\end{aligned}
$$

This permits to rewrite equation (76) in the form of equations for average reduced description parameters $\eta_{a}(t)$ and the generating functional $\mathcal{G}(g(t), u)$ for their correlations $g_{a_{1} \ldots a_{s}}(t)[14]$

$$
\begin{aligned}
\partial_{t} \mathcal{G}(g(t), u) & =\left.\left\{e^{\mathcal{G}\left(g(t), u+\frac{\partial}{\partial \eta}\right)} e^{-\mathcal{G}(g(t), u)}-e^{\mathcal{G}\left(g(t), \frac{\partial}{\partial \eta}\right)}\right\} \sum_{a} u_{a} L_{a}(\eta)\right|_{\eta=\eta(t)}, \\
\partial_{t} \eta_{a}(t) & =\left.e^{\mathcal{G}\left(g(t), \frac{\partial}{\partial \eta}\right)} L_{a}(\eta)\right|_{\eta=\eta(t)} .
\end{aligned}
$$

According to (8), a statistical operator $\rho\left(\eta\left(t, \eta_{0}\right)\right)$ satisfies the Liouville equation

$$
\partial_{t} \rho\left(\eta\left(t, \eta_{0}\right)\right)=\mathbf{L} \rho\left(\eta\left(t, \eta_{0}\right)\right), \quad(t \geqslant 0)
$$

and its average value

$$
\tilde{\rho}(f(t)) \equiv \overline{\rho\left(\eta\left(t, \eta_{0}\right)\right)}, \quad \tilde{\rho}(f(t))=\left.\mathcal{F}\left(f(t), \frac{\partial}{\partial \eta}\right) \rho(\eta)\right|_{\eta=0} \equiv \rho(\eta(t), g(t))
$$

is a solution of this equation too

$$
\partial_{t} \rho(\eta(t), g(t))=\mathbf{L} \rho(\eta(t), g(t)), \quad(t \geqslant 0), \quad \rho(\eta, g) \equiv e^{\mathcal{G}\left(g, \frac{\partial}{\partial \eta}\right)} \rho(\eta) .
$$

The statistical operator $\rho(\eta(t), g(t))$ satisfies the Liouville equation, depends on time through variables $\eta_{a}(t), g_{a_{1} \ldots a_{s}}(t)$, and, therefore, gives a reduced description of the system. Note that this theory introduces correlations $g_{a_{1} \ldots a_{s}}\left(t, \rho_{0}\right)$ as additional ones to $\eta_{a}\left(t, \rho_{0}\right)$ independent reduced description parameters.

Let us consider the relation of the built reduced description to the corresponding one based on the functional hypothesis. Reduced description parameters can be expressed through statistical operator $\rho(\eta(t), g(t))$ by means of projection operator $\mathbf{P}_{a_{1} \ldots a_{s}}$ which destroys correlations of variables $\hat{\eta}_{a}^{(n)} \equiv(-\mathbf{L})^{n} \hat{\eta}_{a}$ in the state described by the statistical operator $\rho(\eta)$

$$
\mathbf{P}_{a \ldots a^{\prime}} \operatorname{Sp} \rho(\eta) \hat{\eta}_{a}^{(n)} \ldots \hat{\eta}_{a^{\prime}}^{\left(n^{\prime}\right)}=\operatorname{Sp} \rho(\eta) \hat{\eta}_{a}^{(n)} \cdot \ldots \cdot \operatorname{Sp} \rho(\eta) \hat{\eta}_{a^{\prime}}^{\left(n^{\prime}\right)} .
$$


This and the relations (7), (80) lead to the formula

$$
\mathbf{P}_{a_{1} \ldots a_{s}} \operatorname{Sp} \tilde{\rho}(f) \hat{\eta}_{a_{1}} \ldots \hat{\eta}_{a_{s}}=f_{a_{1} \ldots a_{s}}
$$

to be considered as a definition of reduced description parameters $f_{a_{1} \ldots a_{s}}(t)$. So, parameters $g_{a_{1} \ldots a_{s}}(t)$ describe correlations which are absent in a state with statistical operator $\rho\left(\eta\left(t, \eta_{0}\right)\right)$. Of course, there is a problem of non-commutativity of operators $\hat{\eta}_{a}$ corresponding to reduced description parameters $\eta_{a}^{( \pm)}(t)$. It is not important in applying this theory to hydrodynamics and kinetics because operators $\hat{\eta}_{a}$ commutate in expressions under the action of the introduced projection operator $\mathbf{P}_{a_{1} \ldots a_{s}}$. Therefore, we shall not pay attention to this and assume commutativity of operators $\hat{\eta}_{a}$. Note also that a similar projection operator was introduced in [14] for hydrodynamics.

The obtained reduced description can be constructed based on the functional hypothesis

$$
\rho(t) \underset{\tau \gg \tau_{0}}{\longrightarrow} \tilde{\rho}\left(f\left(t, \rho_{0}\right)\right)
$$

with reduced description parameters $f_{a_{1} \ldots a_{s}}(t)$ defined by the formula $(83)$. This leads to the same theory because in the considered model (15) the solution of the equation for the statistical operator $\tilde{\rho}(f))$ is unique. Expression (81) for the statistical operator $\rho(\eta, g)$ via the operator $\rho(\eta)$ suggests the following expression for a quasi-equilibrium statistical operator in the presence of nonequilibrium correlations [14]

$$
\rho_{q}(\eta, g)=e^{\mathcal{G}\left(g, \frac{\partial}{\partial \eta}\right)} \rho_{q}(Y(\eta)) .
$$

This permits to argue that similar to (11) the relations

$$
g_{a_{1} \ldots a_{s}}\left(0, \rho_{q}(\eta, g)\right)=g_{a_{1} \ldots a_{s}}, \quad \eta_{a}\left(0, \rho_{q}(\eta, g)\right)=\eta_{a}
$$

are true. Here $\eta_{a}\left(0, \rho_{0}\right), g_{a_{1} \ldots a_{s}}\left(0, \rho_{0}\right)$ are the effective initial conditions for the reduced description parameters $\eta_{a}\left(t, \rho_{0}\right), g_{a_{1} \ldots a_{s}}\left(t, \rho_{0}\right)$.

\section{Reduced description taking into account binary correlations and the generalized Ernst and Dorfman approach}

It is assumed here that there are only binary correlations $g_{a b}(t)$ while more complicated correlations are absent. In this situation equations for the reduced description parameters take the form

$$
\begin{aligned}
\partial_{t} g_{a b}(t) & =\left.e^{\mathcal{G}_{2}\left(g(t), \frac{\partial}{\partial \eta}\right)} \sum_{c}\left\{g_{a c}(t) \frac{\partial L_{b}(\eta)}{\partial \eta_{c}}+g_{b c}(t) \frac{\partial L_{a}(\eta)}{\partial \eta_{c}}\right\}\right|_{\eta=\eta(t)}, \\
\partial_{t} \eta_{a}(t) & =\left.e^{\mathcal{G}_{2}\left(g(t), \frac{\partial}{\partial \eta}\right)} L_{a}(\eta)\right|_{\eta=\eta(t)}, \quad\left(\mathcal{G}_{2}(g, u) \equiv \frac{1}{2} \sum_{a b} g_{a b} u_{a} u_{b}\right) .
\end{aligned}
$$

According to $(15),(19)$, and (83), there are no equilibrium correlations $\stackrel{o}{g}_{a b}=0$ because $\left.\tilde{\rho}(f)=\stackrel{o}{f}\right)=$

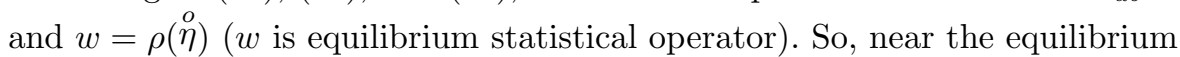

$$
\eta_{a}^{( \pm)}(t)=\stackrel{o}{\eta}_{a}+\delta \eta_{a}^{( \pm)}(t)+O\left(g^{2}\right), \quad g_{a b}^{( \pm)}(t)=\delta g_{a b}^{( \pm)}(t)+O\left(g^{2}\right)
$$

where we return to the description of the evolution in two directions.

Close to equilibrium, statistical operator of the system (81) has the form

$$
\begin{aligned}
\rho^{( \pm)}\left(\eta^{( \pm)}(t), g^{( \pm)}(t)\right) & =w+\delta \rho^{( \pm)}(t)+O\left(g^{2}\right) \\
\delta \rho^{( \pm)}(t) & =\sum_{a} \rho_{a}^{( \pm)} \delta \eta_{a}^{( \pm)}(t)+\sum_{a b} \rho_{a b}^{( \pm)} \delta g_{a b}^{( \pm)}(t)
\end{aligned}
$$


where operators $\rho_{a}^{( \pm)}$were introduced in (19) and $\rho_{a b}^{( \pm)}$is given by the formula

$$
\rho_{a b}^{( \pm)}=\left.\frac{1}{2} \frac{\partial \rho^{( \pm)}(\eta)}{\partial \eta_{a} \partial \eta_{b}}\right|_{\eta=\eta} .
$$

Time equations for variables (88) can be written as follows

$$
\begin{aligned}
& \partial_{t} \delta \eta_{a}^{( \pm)}(t)=\sum_{b} M_{a b}^{( \pm)} \delta \eta_{b}^{( \pm)}(t)+\sum_{b c} M_{a, b c}^{( \pm)} \delta g_{a b}^{( \pm)}(t), \\
& \partial_{t} \delta g_{a b}^{( \pm)}(t)=\sum_{c}\left\{M_{a c}^{( \pm)} \delta g_{c b}^{( \pm)}(t)+M_{b c}^{( \pm)} \delta g_{c a}^{( \pm)}(t)\right\},
\end{aligned}
$$

where the matrix $M_{a b}^{( \pm)}$was introduced in (21) and $M_{a, b c}^{( \pm)}$is defined by the relation

$$
M_{a, b c}^{( \pm)}=\operatorname{Sp} \hat{\dot{\eta}}_{a} \rho_{b c}^{( \pm)} .
$$

The definition (83) of the reduced description parameters shows that

$$
\delta \eta_{a}^{( \pm)}(t)=\operatorname{Sp} \delta \rho^{( \pm)}(t) \hat{\eta}_{a}, \quad \delta g_{a b}^{( \pm)}(t)=\mathbf{P}_{a b} \operatorname{Sp} \delta \rho^{( \pm)}(t) \delta \hat{\eta}_{a} \delta \hat{\eta}_{b}, \quad\left(\delta \hat{\eta}_{a} \equiv \hat{\eta}_{a}-\stackrel{o}{\eta}_{a}\right) .
$$

Statistical operators $\rho_{a}^{( \pm)}, \rho_{a b}^{( \pm)}$have the properties

$$
\begin{aligned}
\mathbf{P}_{a b} \operatorname{Sp} \rho_{c}^{( \pm)} \delta \hat{\eta}_{a} \delta \hat{\eta}_{b} & =0, \quad \operatorname{Sp} \rho_{a}^{( \pm)} \hat{\eta}_{b}=\delta_{a b}, \quad \operatorname{Sp} \rho_{a b}^{( \pm)} \hat{\eta}_{c}=0, \\
\mathbf{P}_{a b} \operatorname{Sp} \rho_{c d}^{( \pm)} \delta \hat{\eta}_{a} \delta \hat{\eta}_{b} & =\frac{1}{2}\left(\delta_{a c} \delta_{b d}+\delta_{b c} \delta_{a d}\right)
\end{aligned}
$$

following from (19), (90) and the projection operator $\mathbf{P}_{a \ldots a^{\prime}}$ definition (82).

Functional hypothesis (84) near the equilibrium (according to (89) and (91)) leads to the asymptotic relation

$$
\begin{array}{r}
e^{t \mathbf{L}} \delta \rho_{0} \underset{ \pm t \gg \tau_{0}}{\longrightarrow} \sum_{a b} \rho_{a}^{( \pm)} e_{a b}^{t M^{( \pm)}} \delta \eta_{b}^{( \pm)}\left(0, \delta \rho_{0}\right)+\sum_{a b a^{\prime} b^{\prime}} \rho_{a b}^{( \pm)} e_{a a^{\prime}}^{t M M^{( \pm)}} e_{b b^{\prime}}^{t M^{( \pm)}} \delta g_{a^{\prime} b^{\prime}}^{( \pm)}\left(0, \delta \rho_{0}\right) \\
+\sum_{a b c d a^{\prime} b^{\prime}} \int_{0}^{t} \mathrm{~d} t^{\prime} \rho_{a}^{( \pm)} e_{a b}^{\left(t-t^{\prime}\right) M^{( \pm)}} M_{b, c d}^{( \pm)} e_{c a^{\prime}}^{t^{\prime} M^{( \pm)}} e_{d b^{\prime}}^{t^{\prime} M^{( \pm)}} \delta g_{a^{\prime} b^{\prime}}^{( \pm)}\left(0, \delta \rho_{0}\right),
\end{array}
$$

which is similar to $(22)$. Here $\delta \eta_{a}^{( \pm)}\left(0, \rho_{0}\right), \delta g_{a b}^{( \pm)}\left(0, \rho_{0}\right)$ are effective initial conditions for parameters $\delta \eta_{a}^{( \pm)}\left(t, \rho_{0}\right), \delta g_{a b}^{( \pm)}\left(t, \rho_{0}\right)$. Choosing initial state $\delta \rho_{0}$ in the form (17), we obtain the following expression for asymptotics of time correlation function $K_{A B}(t)$

$$
\begin{aligned}
K_{A B}^{( \pm)}(t)= & \sum_{a b} \operatorname{Sp} \hat{A} \rho_{a}^{( \pm)} e_{a b}^{t M^{( \pm)}} \mathbf{C}_{b}^{( \pm)}(\hat{B})+\sum_{a b c d} \operatorname{Sp} \hat{A} \rho_{a b}^{( \pm)} e_{a c}^{t M^{( \pm)}} e_{b d}^{t M^{( \pm)}} \mathbf{C}_{c d}^{( \pm)}(\hat{B}) \\
& +\sum_{a b c d a^{\prime} b^{\prime}} \int_{0}^{t} \mathrm{~d} t^{\prime} \operatorname{Sp} \hat{A} \rho_{a}^{( \pm)} e_{a b}^{\left(t-t^{\prime}\right) M^{( \pm)}} M_{b, c d}^{( \pm)} e_{c a^{\prime}}^{t^{\prime} M^{( \pm)}} e_{d b^{\prime}}^{t^{\prime} M^{( \pm)}} \mathbf{C}_{a^{\prime} b^{\prime}}^{( \pm \hat{B})} .
\end{aligned}
$$

Here, similar to (25), memory functions

$$
\mathbf{C}_{a}^{( \pm)}(\hat{A})=\delta \eta_{a}^{( \pm)}(0, w \chi(\hat{A})), \quad \mathbf{C}_{a b}^{( \pm)}(\hat{A})=\delta g_{a b}^{( \pm)}(0, w \chi(\hat{A}))
$$

are introduced. The first term in expression (96) coincides with (24), the second and the third ones take binary correlations into account.

The formula (96) can be applied to the investigation of correlation and Green functions within the framework of the generalized Ernst and Dorfman approach. In their theory only the second 
term in (96) was taken into account [1]. For the first time this term as a leading contribution to (96) was proposed by Kadanoff and Swift [4] within the framework of a mode-mode coupling theory. Therefore, our expression for asymptotics (96) can be considered as a generalization of the mode-mode coupling theory. In hydrodynamics the second and the third terms from (96) were taken into consideration in the generalized Ernst and Dorfman equation (69) in [16]. Solution of the last equation was studied in [17] with an application to the calculation of kinetic coefficients of a fluid.

Of course, all the values entering (96) can be calculated. Really, values $\rho_{a}^{( \pm)}, \mathbf{C}_{a}^{( \pm)}(\hat{A}), M_{a b}^{( \pm)}$ were calculated in the generalized perturbation theory in sections 6 (see formulae (61) and (64)). So, we have to calculate statistical operator $\rho_{a b}^{( \pm)}$, memory function $\mathbf{C}_{a b}^{( \pm)}(\hat{A})$ and matrix $M_{a, b c}^{( \pm)}$. Equation (14) and definition (90) give the following integral equation for $\rho_{a b}^{( \pm)}$

$$
\rho_{a b}^{( \pm)}=\rho_{a b}^{q( \pm)}+\int_{0}^{ \pm \infty} \mathrm{d} \tau e^{\tau \mathbf{L}}\left\{\mathbf{L} \rho_{a b}^{q( \pm)}-\sum_{c} \rho_{c}^{( \pm)} M_{c, a b}^{( \pm)}-\sum_{c} \rho_{a c}^{( \pm)} M_{c b}^{( \pm)}-\sum_{c} \rho_{b c}^{( \pm)} M_{c a}^{( \pm)}\right\}
$$

where the quasi-equilibrium statistical operator

$$
\rho_{a b}^{q( \pm)}=\frac{1}{2} w\left\{\sum_{c} \chi\left(w, \hat{\eta}_{c}\right) B_{c, a b}^{( \pm)}+\sum_{c d} \chi\left(w, \delta \hat{\eta}_{c} \delta \hat{\eta}_{d}\right) A_{c a}^{( \pm)-1} A_{d b}^{( \pm)-1}\right\}
$$

is introduced (taking into account (33) and definition $\left.B_{a, b c}^{( \pm)} \equiv \frac{\partial Y_{a}^{( \pm)}(\eta)}{\partial \eta_{b} \partial \eta_{c}}\right|_{\eta=\eta}$ ). Equation (98) should be solved with respect to statistical operator $\rho_{a b}^{( \pm)}$and matrices $B_{a, b c}^{( \pm)}, M_{a, b c}^{( \pm)}$using (92) and the third formula in (94). In order to construct the generalized perturbation theory for these values the integral equation (98) has to be transformed (see transformation of (31) to (51)).

According to (96), the asymptotics of important time correlation functions are expressed through matrices

$$
A_{a b, c}^{( \pm)}=\mathbf{C}_{a b}^{( \pm)}\left(\hat{\eta}_{c}\right), \quad A_{a, b c}^{( \pm)}=\mathbf{C}_{a}^{( \pm)}\left(\delta \hat{\eta}_{b} \delta \hat{\eta}_{c}\right), \quad A_{a b, c d}^{( \pm)}=\mathbf{C}_{a b}^{( \pm)}\left(\delta \hat{\eta}_{c} \delta \hat{\eta}_{d}\right)
$$

similarly to (26). The relations (86) and formula (99) lead to the following expressions for these matrices

$$
A_{a b, c d}^{( \pm)}=A_{a c}^{( \pm)} A_{b d}^{( \pm)}+A_{a d}^{( \pm)} A_{b c}^{( \pm)}, \quad A_{a, b c}^{( \pm)}=\sum_{d a^{\prime} b^{\prime}} A_{a d}^{( \pm)} B_{d, a^{\prime} b^{\prime}}^{( \pm)} A_{a^{\prime} b}^{( \pm)} A_{b^{\prime} c}^{( \pm)}, \quad A_{a b, c}^{( \pm)}=0 .
$$

The last formula shows that the matrix $B_{a, b c}^{( \pm)}$calculation can be also done based on our results in section 6 , where the matrix $A_{a b}^{( \pm)}$and memory function $\mathbf{C}_{a}^{( \pm)}(\hat{A})$ were found (see formulae (61), (64)).

The memory function $\mathbf{C}_{a b}^{( \pm)}(\hat{A})$ for an arbitrary operator $\hat{A}$ can be calculated taking into account reversibility of the system. Formula (96) gives a relation

$$
\sum_{a} \operatorname{Sp} \rho_{a}^{(+)} \hat{A} \mathbf{C}_{a}^{(+)}(\hat{B})+\sum_{a b} \operatorname{Sp} \rho_{a b}^{(+)} \hat{A} \mathbf{C}_{a b}^{(+)}(\hat{B})=\sum_{a} \operatorname{Sp} \rho_{a}^{(-)} \hat{B} \mathbf{C}_{a}^{(-)}(\hat{A})+\sum_{a b} \operatorname{Sp} \rho_{a b}^{(-)} \hat{B} \mathbf{C}_{a b}^{(-)}(\hat{A}),(10
$$

which is similar to (29). Substituting $\hat{\eta}_{c}$ in this relation instead of $\hat{A}$ and using formulae (94) and (101) confirm the formula (26). Substituting $\delta \hat{\eta}_{c} \delta \hat{\eta}_{d}$ in this relation instead of $\hat{A}$, applying the operator $\mathbf{P}_{c d}$, and using formulae (94) and (101) lead to an expression for the memory function of correlations

$$
\mathbf{C}_{a b}^{( \pm)}(\hat{A})=2 \sum_{c d} \operatorname{Sp} \hat{A} \rho_{c d}^{(\mp)} A_{c a}^{(\mp)} A_{d b}^{(\mp)}
$$

(in hydrodynamics this result was obtained in [11]). 
So, all the values in the expression (96) for asymptotics $K_{A B}^{( \pm)}$of the correlation functions can be calculated based on the developed theory. The results take the simplest form in the case of using variables $\hat{\eta}_{n}$, defined in (49) instead of $\hat{\eta}_{a}$. For example, in order to calculate corrections to the Ernst and Dorfman theory for the correlation function $D_{n n^{\prime}}(z)$ (see $(68)$ ) it is enough to use the leading order contribution to corresponding values

$$
\begin{aligned}
\rho_{n n^{\prime}}^{( \pm)} & =\frac{1}{2} w \chi\left(w, \mathbf{Q} \delta \hat{\eta}_{n}^{+} \delta \hat{\eta}_{n^{\prime}}^{+}\right)+\cdots, & M_{n, n^{\prime} n^{\prime \prime}}^{( \pm)} & =\frac{1}{2}\left(\hat{I}_{n}, \delta \hat{\eta}_{n^{\prime}}^{+} \delta \hat{\eta}_{n^{\prime \prime}}^{+}\right)+\cdots, \\
\mathbf{C}_{n n^{\prime}}^{( \pm)}(\hat{A}) & =\left(\delta \hat{\eta}_{n} \delta \hat{\eta}_{n^{\prime}}, \mathbf{Q} \hat{A}\right)+\cdots, & A_{n, n^{\prime} n^{\prime \prime}}^{( \pm)} & =\left(\delta \hat{\eta}_{n}, \delta \hat{\eta}_{n^{\prime}}^{+} \delta \hat{\eta}_{n^{\prime \prime}}^{+}\right)+\cdots
\end{aligned}
$$

(operators $\mathbf{Q}$ and $\hat{I}_{n}$ are defined in $(62)$ ).

For the first time the ideas considered in this section were developed in hydrodynamics in $[16,17]$.

\section{Conclusions}

Discussion of the Bogolyubov reduced description method based on the functional hypothesis shows that it can be a ground for the theory of nonequilibrium processes. This theory permits to develop a general approach to the investigation of time correlation and Green functions. Solution of the problem of convergence of contributions of a perturbation theory to basic values of the theory can be reduced to an investigation of peculiarities of correlation functions. The corresponding approach is based on the generalized perturbation theory with partial summation of contributions of the usual perturbation theory. Contributions of the generalized theory exist due to the procedure of the natural regularization of an integral equation for the statistical operator of the system. The generalized perturbation theory introduces correlation functions, which can be studied based on the generalized Ernst and Dorfman theory proposed in the paper. This theory permits to build asymptotic expansions of correlation functions in a small parameter and, therefore, of the basic values of the reduced description. These expansions are non-analytic in general. In this way, peculiarities of Green functions can be also studied because they are expressed through correlation functions.

This work was supported by the State Foundation for Fundamental Research of Ukraine (project No. 2.7/418).

\section{References}

1. Ernst M.H., Dorfman J.R. Nonanalytic dispersion relations.II. The general fluid, J.Stat.Phys., 1975, 12, No. 4, 311-359.

2. Zwanzig R. Ensemble method in the theory of irrevesibility, J. Chem.Phys., 1960, 33, No. 5, 1338-1341.

3. Mori H. Transport, collective motion and Brownian motion, Progr. Theor. Phys., 1965, 33, 423-433.

4. Kadanoff L.P., Swift J. Transport coefficients near the liquid-gas critical point, Phys. Rev., 1968, 166, No. 1, 89-101.

5. Bogolyubov N.N. Problems of Dynamical Theory in Statistical Physics. Moscow-Leningrad: Gostekhizdat, 1946, 119 (in Russian).

6. Akhiezer A.I., Peletmisky S.V. Methods of Statistical Physics. Moscow: Nauka, 1977, 386 (in Russian); Oxford: Pergamon Press, 1981.

7. Zubarev D.N., Morozov V.G., Roepke G. Statistical Mechanics of Nonequilibrium Processes, Moscow: Fizmatlit, 2002, 1, 431, 2, 296 (in Russian).

8. Kovalevsky M.Yu., Peletminsky S.V., Sokolovsky A.I. Nonequilibrium entropy and principle of symmetry of kinetic coefficients, Theor. Mat. Fiz., 1977, 33, No. 3, 377-389 (in Russian).

9. Grad H. Asymptotic theory of the Boltzman equation, Phys. Fluids, 1963, 6, No. 2, 147-181.

10. Peletminsky S.V. Functional hypothesis and low frequency asymptotics of the Green functions. In: Problems of Modern Theoretical Physics, Kiev: Naukova dumka, Ed. V.G. Bar'yakhtar, S.V. Peletminsky, 154-167 (in Russian).

11. Sokolovsky A.I. Effective initial conditions for equations of fluctuation hydrodynamics, Dopovidi Nats. Akad. Nauk Ukrainy, 1998, No. 5, 83-88 (in Russian). 
12. Balescu R. Equilibrium and Nonequilibrium Statistical Mechanics, Moscow: Mir, 1978, 1, 406 , 2, 400 (in Russian), Wiley, New York, 1975.

13. Sokolovsky A.I. Equations of hydrodynamics close to equilibrium in the absence of long correlations, Ukr. Fiz. Zhurn., 1992, 37, No. 10, 1528-1536 (in Russian).

14. Peletminsky S.V., Sokolovsky A.I. General equations of fluctuation hydrodynamics, Ukr. Fiz. Zhurn., 1992, 37, No. 10, 1521-1528 (in Russian).

15. Peletminsky S.V., Slusarenko Yu.V.Stochastic derivation of kinetic and hydrodynamic equations of long-wave fluctuations, Ukr. Fiz. Zhurn., 1994, 39, No. 1, 112-119 (in Ukrainian).

16. Sokolovsky A.I., Sokolov P.G. Integral equation for flux-flux correlation functions and kinetic coefficients of a fluid, Visnyk of Dnipropetrovs'k University, Physics, Radio Electronics, 1998, 1, No. 3, 76-82 (in Russian).

17. Sokolovsky A.I. Influence of fluctuations on dynamics of a fluid, Visnyk of Dnipropetrovs'k University, Physics, Radio Electronics, 1998, 1, No. 3, 64-75 (in Russian).

\title{
Скорочений опис нерівноважних процесів та кореляційні Функції. Розбіжності та неаналітичність
}

\author{
А.І.Соколовський \\ Дніпропетровський національний університет, вул. Наукова 13, Дніпропетровськ, Україна, 49050 \\ Отримано 16 травня 2006 р., в остаточному вигляді - 6 липня 2006 р. \\ На основі методу скороченого опису Боголобова, що ґрунтується на функціональній гіпотезі, роз- \\ вивається повна теорія для дослідження часових кореляційних функцій. Обговорюється проблема \\ розбіжностей в теорії нерівноважних процесів та її зв'язок з неаналітичною залежністю основних \\ величин теорії від малого параметра теорії збурень. Пропонується природна регуляризація інте- \\ гральних рівнянь теорії. В рамках моделі повільних змінних (гідродинаміка рідини, кінетика газу) \\ конструюється узагальнена теорія збурень, яка відповідає частковому сумуванню у звичайній теорії \\ збурень. Властивості функцій Гріна обговорюються на основі формалізму резольвенти оператора \\ Ліувілля. Розробляється узагальнена теорія Ернста та Дорфмана, яка дозволяє вивчати особливо- \\ сті кореляційних функцій і функцій Гріна та розв'язувати проблему збіжності у методі скороченого \\ опису.
}

Ключові слова: скорочений опис нерівноважних процесів, функціональна гіпотеза, проблема збіжності, природна регуляризація, асимптотика часових кореляційних функцій, функції Гріна, особливості кореляційних функцій і функцій Гріна, узагальнена теорія Ернста та Дорфмана

PACS: 05.20.Dd, 05.30-d 